\title{
Re: Fertility Outcomes After Extended Searches for Ejaculated Spermatozoa in Men with Virtual Azoospermia
}

\author{
Miller N , Biron-Shental T², Pasternak $\mathrm{Y}^{3}$, Belenky $\mathrm{M}^{4}$, Shefi $\mathrm{S}^{5}$, Itsykson $\mathrm{P}^{6}$, Berkovitz $\mathrm{A}^{7}$ \\ ${ }^{1}$ Meir Medical Center, Department of Obstetrics and Gynecology, Kfar-Saba, Israel \\ ${ }^{2}$ Meir Medical Center, Department of Obstetrics and Gynecology, Kfar-Saba, Israel; Tel Aviv University Sakler Faculty of Medicine, Tel Aviv, Israel \\ ${ }^{3}$ Meir Medical Center, Department of Obstetrics and Gynecology, Kfar-Saba, Israel \\ ${ }^{4}$ Male Fertility Center, Rishon LeZion, Israel \\ ${ }^{5}$ Assuta Medical Center, Clinic of Advanced Andrology, Tel Aviv, Israel \\ ${ }^{6}$ Assuta Medical Center, Tel Aviv, Israel \\ ${ }^{7}$ Meir Medical Center, Department of Obstetrics and Gynecology, Kfar-Saba, Israel; Tel Aviv University Sakler Faculty of Medicine, Tel Aviv, Israel; \\ Assuta Medical Center, Tel Aviv, Israel
}

Fertil Steril 2017;107:1305-1311. doi: 10.1016/j.fertnstert.2017.04.005.

\section{EDITORIAL COMMENT}

Based on the World Health Organization 2010 recommendation, seminal fluid should be centrifuged at $3000 \mathrm{~g}$ for $15 \mathrm{minutes}$ and the pellet should be detected for the presence of spermatozoa. If any sperm is observed in the pellet, the patient is considered as having cryptozoospermia. In this study, the investigators identified a group of men with cryptozoospermia and they searched centrifuged pellet around 2 to 4 hours to find sperm and called this group "virtual azoospermia" if they found a few. Using ejaculated sperm and testicular sperm for intracytoplasmic sperm injection, fertilization rates (56\% vs 50\%) were similar between the groups. Although the pregnancy rate was higher in the ejaculated group (64\%) than in microsurgical testicular sperm extraction (microTESE) group (50\%), it was not statistically significant. "Take-home baby" rates were higher in microTESE group (82\%) compared to ejaculated sperm group (58\%) but not statistically significant. This study shows that in vitro fertilization laboratory technicians have a very important role in extended spermatozoa search in the ejaculate as well as urologists working under the microscope to find viable sperm within the testes. In addition, finding motile sperm in ejaculate with extensive search, rescue the patient from surgical sperm recovery. However, this condition has no effect on the rates of pregnancy and live birth but significantly on high miscarriage rate in the first trimester (52\% vs $8.6 \%$ ).

Emre Bakırcıoğlu, MD

बCopyright 2018 by the Association of Urological Surgery / Journal of Urological Surgery published by Galenos Publishing House. 